\title{
Improved survival with pulmonary metastasectomy: An analysis of 1720 patients with pulmonary metastatic melanoma
}

Rebecca P. Petersen, MD, MSc, ${ }^{a}$ Steven I. Hanish, MD, ${ }^{a}$ John C. Haney, MD, ${ }^{a}$ Charles C. Miller III, PhD, ${ }^{b}$

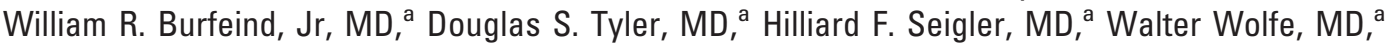

Thomas A. D'Amico, MD, and David H. Harpole, Jr, MD ${ }^{a}$

Earn CME credits at http:// cme.ctsnetjournals.org

Supplemental material is available online.
From the Department of Surgery, Duke University Medical Center, ${ }^{\mathrm{a}}$ Durham, NC, and the Department of Cardiothoracic and Vascular Surgery, University of TexasHouston Health Science Center, ${ }^{\mathrm{b}}$ Houston, Texas.

Read at the Eighty-sixth Annual Meeting of The American Association for Thoracic Surgery, Philadelphia, Pa, April 29-May 3, 2006.

Received for publication April 28, 2006; revisions received July 18, 2006; accepted for publication Aug 7, 2006.

Address for reprints: David H. Harpole, Jr, MD, Department of Surgery, Duke University Medical Center, Box 3617, Durham, NC 27710 (E-mail: harpo002@mc.duke.edu).

J Thorac Cardiovasc Surg 2007;133:104-10 $0022-5223 / \$ 32.00$

Copyright (๑) 2007 by The American Association for Thoracic Surgery

doi:10.1016/j.jtcvs.2006.08.065
Objectives: The outcomes of patients with metastatic melanoma are poor. Although prognostic models have been developed to predict the occurrence of pulmonary metastasis from cutaneous melanoma, few data exist to define the outcomes of these patients once metastasis has occurred. The objective of this study was to discriminate predictors of survival for patients with pulmonary metastatic melanoma.

Methods: We found 1720 patients with pulmonary metastasis listed in a prospective comprehensive cancer center database of 14,057 consecutive patients with melanoma (Jan 1, 1970-June 1, 2004). Demographic and histopathologic data, time and location of recurrences, number of pulmonary nodules, and subsequent therapies were collected. Univariate and multivariate Cox proportional hazards models were used to identify predictors of survival for patients with pulmonary metastatic melanoma.

Results: The median survival was 7.3 months after development of pulmonary metastasis. Significant predictors of survival from the multivariate model included nodular histologic type $(P=.033)$, disease-free interval $(P<.001)$, number of pulmonary metastases $(P=.012)$, presence of extrathoracic metastasis $(P<.001)$, and performance of pulmonary metastasectomy $(P<.001)$. Interactions were identified between metastasectomy and disease-free interval and presence of extrathoracic metastasis. Surgery was associated with a survival advantage of 12 months for patients with a disease-free interval longer than 5 years (19 vs 7 months, $P<.01)$ and of 10 months for patients without extrathoracic metastasis $(18$ vs 8 months, $P<.01$ ).

Conclusions: When all other identified risk factors were controlled for mathematically, metastasectomy maintained a significant survival advantage for patients with pulmonary metastatic melanoma. These data support the role of surgery for a select subset of patients with pulmonary metastasis.

I n 2004, approximately 55,100 people in the United States were diagnosed with cutaneous melanoma, and the incidence only continues to rise. ${ }^{1}$ Approximately $30 \%$ of patients with cutaneous melanoma have metastatic disease develop, resulting in a dismal prognosis with a median survival of only 6 to 8 months and 5 -year survival of less than $5 \% .^{2,3}$ More than $80 \%$ of patients with metastatic melanoma initially show only one distant organ site involved, most commonly the lung. ${ }^{3-5}$ Approximately $40 \%$ of patients with metastatic melanoma initially show isolated pulmonary metastasis. ${ }^{3}$

Once distant metastasis has developed, the treatment options remain limited. This is largely due to the failure of numerous systemic therapies, including chemotherapy, immunotherapy, and more recently molecular targeted agents, to improve 


\section{Abbreviations and Acronyms}

$\mathrm{CT}=$ computed tomography

PET $=$ positron emission tomography overall survival in patients with stage IV disease. Surgical therapy, however, has been shown in several studies to be associated with a 5-year survival as high as $39 \%,{ }^{6}$ as opposed to a $3 \%$ to $5 \%$-year survival for nonsurgically treated patients. ${ }^{2,7-10}$ These findings suggest that a select subset of patients with pulmonary metastatic melanoma may benefit from metastasectomy. Several large studies of patients with pulmonary metastatic disease have determined prognostic factors for survival, but these have included patients with a variety of primary tumor types and have failed to discriminate melanoma-specific prognostic factors. The objective of this study was to discriminate predictors of survival in patients with pulmonary metastatic melanoma.

\section{Patients and Methods}

\section{Patient Population}

This study was reviewed and approved by the Duke institutional review board. Among the 14,057 patients with primary melanoma who were initially evaluated at the Duke Comprehensive Cancer Center between January 1, 1970, and December 31, 2004, we found 1720 patients with metastatic pulmonary melanoma. The database was prospectively maintained, and baseline demographic data, time and location of primary tumor, histopathologic data, subsequent therapies (immunotherapy, chemotherapy, nodal dissections, and other resections), overall and cancer-specific survivals, and time and location of metastases were available for all patients. All patients with primary melanoma were followed up by clinical examination every 6 months for 5 years after the initial diagnosis and then annually thereafter. In addition, annual surveillance chest radiography was routinely performed for all patients to detect pulmonary metastasis.

\section{Statistical Methods}

Univariate and multivariate regression analyses with a Cox proportional hazards model were performed to examine the relationship between potential prognostic factors and overall survival for patients with metastatic pulmonary melanoma. The following variables were analyzed: age, sex, race, primary site, histologic type, Clark level, Breslow thickness, ulceration of the primary lesion, regional lymph node status, number of pulmonary metastases, disease-free interval between initial diagnosis and development of pulmonary metastasis, presence of extrathoracic metastasis, subsequent therapies (immunotherapy and chemotherapy) and performance of pulmonary metastasectomy. Predictor variables identified in the univariate analysis with a $P$ value less than .1 were further examined in a multiple Cox proportional hazards model.

Overall survival was defined as the time between the initial diagnosis of pulmonary metastasis and the date of last follow-up or death. Probabilities for development of pulmonary metastasis and overall survival were estimated with the Kaplan-Meier method.
Kaplan-Meier survival analyses stratifying on significant predictor variables identified in the multivariate analysis were also performed, and differences were tested with the log-rank test. Analyses were carried out with Intercooled STATA version 9.0 software (Stata Corporation, College Station, Tex).

\section{Results}

The estimated risks of development of pulmonary metastasis for the entire melanoma population $(\mathrm{n}=13,565)$ were $13 \%$ at 5 years, $17 \%$ at 10 years, and $23 \%$ at 20 years after initial diagnosis (Figure E1). Patients who had unknown primary tumors or who had pulmonary metastasis at initial presentation were excluded from this analysis $(n=492)$. The median follow-up of living patients was 65 months (0.1-524 months).

The overall survivals for patients with pulmonary metastatic melanoma $(\mathrm{n}=1720)$ were $34 \%$ at 1 year, $14 \%$ at 2 years, and $6 \%$ at 5 years (Figure E2). Patient demographic and melanoma histopathologic characteristics are displayed in Table 1. The most common method of diagnosis for pulmonary metastatic melanoma was chest radiography (n $=774$ ). The remaining cases were diagnosed by chest computed tomography $(\mathrm{CT}, \mathrm{n}=410)$, surgery $(\mathrm{n}=318)$, transthoracic needle aspiration $(n=192)$, bronchoscopic biopsy $(n=18)$, and autopsy $(n=8)$. For patients undergoing surgery, the most common approach was unilateral thoracotomy $(\mathrm{n}=255)$, followed by thoracoscopic surgery $(n=40)$ and bilateral thoracotomy $(n=23)$. The most common type of anatomic resection was stapled wedge $(\mathrm{n}=$ 210), followed by lobectomy ( $\mathrm{n}=96)$, segmentectomy ( $\mathrm{n}$ $=9)$, and pneumonectomy $(\mathrm{n}=3)$.

Among the demographic and histopathologic characteristics, age older than 50 years $(P=.01)$, African American race $(P=.03)$, nodular histologic type $(P=.01)$, Breslow thickness (1-4 mm $P=.01$ and $>4 \mathrm{~mm} P=.002)$, ulceration of the primary lesion $(P=.003)$, positive regional lymph nodes $(P=.001)$, more than 2 pulmonary metastases $(P<.001)$, disease-free interval $(<1$ year $P<.001$ and $1-5$ years $P<.001)$, presence of extrathoracic metastasis $(P<$ $.001)$, and performance of pulmonary metastasectomy $(P<$ $.001)$ were found to be significant univariate predictors of overall survival. When examined in a multivariate model, nodular histologic type, disease-free interval, number of pulmonary metastases, presence of extrathoracic metastasis, and performance of pulmonary metastasectomy were found to be independent predictors of overall survival (Table 2).

Interactions were found between metastasectomy and disease-free interval and presence of extrathoracic metastasis. Among patients with a disease-free interval longer than 5 years, surgery was associated with a survival advantage of 12 months (19 vs 7 months, $P<.01$ ). In patients without evidence of extrathoracic metastasis, pulmonary metastasectomy was associated with a survival advantage of 10 months (18 vs 8 months, $P<.01$; Figure E4). After strat- 
TABLE 1. Comparison of total patients, surgical patients, and nonsurgical patients $(n=1720)$

\begin{tabular}{|c|c|c|c|c|}
\hline Risk factor & All patients $(n=1720)$ & Surgical $(n=318)$ & Nonsurgical $(n=1402)$ & $P$ value \\
\hline \multicolumn{5}{|l|}{ Age (y) } \\
\hline No. $>50 \mathrm{y}$ & $815(47 \%)$ & $150(47 \%)$ & $665(47 \%)$ & .93 \\
\hline Mean $\pm S D$ & $49 \pm 15$ & $47 \pm 15$ & $49 \pm 15$ & .16 \\
\hline Male (No.) & $1091(63 \%)$ & $219(69 \%)$ & $872(62 \%)$ & .02 \\
\hline \multicolumn{5}{|l|}{ Race (No.) } \\
\hline White & $1680(97 \%)$ & $315(94 \%)$ & $1365(97 \%)$ & .01 \\
\hline Black & $38(2 \%)$ & $3(1 \%)$ & $35(2.5 \%)$ & .1 \\
\hline Other & $26(1 \%)$ & $18(5 \%)$ & $8(0.5 \%)$ & $<.001$ \\
\hline \multicolumn{5}{|l|}{ Primary site (No.) } \\
\hline Extremity & $435(25 \%)$ & $73(23 \%)$ & $362(26 \%)$ & .29 \\
\hline Trunk & $678(39 \%)$ & $122(38 \%)$ & $556(40 \%)$ & .67 \\
\hline Head and neck & $386(23 \%)$ & $83(26 \%)$ & $303(22 \%)$ & .08 \\
\hline Mucosal-genital/oral/rectal & $54(3 \%)$ & $5(2 \%)$ & $49(3 \%)$ & .08 \\
\hline Unknown & $167(10 \%)$ & $35(11 \%)$ & $132(9 \%)$ & .39 \\
\hline \multicolumn{5}{|l|}{ Histologic type (No.) } \\
\hline Nodular & $364(21 \%)$ & $59(18 \%)$ & $305(22 \%)$ & .21 \\
\hline Acral lentiginous & $98(6 \%)$ & $7(2 \%)$ & $91(6 \%)$ & .003 \\
\hline Superficial spreading & $678(39 \%)$ & $121(38 \%)$ & $557(40 \%)$ & .58 \\
\hline Lentigo maligna & $63(4 \%)$ & $21(7 \%)$ & $42(3 \%)$ & .002 \\
\hline Other, not otherwise specified & $517(30 \%)$ & $110(35 \%)$ & $407(29 \%)$ & .051 \\
\hline \multicolumn{5}{|l|}{ Clark level (No., $\mathrm{n}=1258$ ) } \\
\hline I-II & $77(6 \%)$ & $23(9 \%)$ & $54(5 \%)$ & .02 \\
\hline III & $386(31 \%)$ & $76(31 \%)$ & $310(31 \%)$ & \\
\hline IV-V & $795(63 \%)$ & $148(60 \%)$ & $647(64 \%)$ & \\
\hline \multicolumn{5}{|l|}{ Thickness (No. $n=1085$ ) } \\
\hline$<1 \mathrm{~mm}$ & $140(13 \%)$ & $43(19 \%)$ & $97(11 \%)$ & .006 \\
\hline $1-4 \mathrm{~mm}$ & $672(62 \%)$ & $134(60 \%)$ & $538(63 \%)$ & \\
\hline$>4 \mathrm{~mm}$ & $273(25 \%)$ & $48(21 \%)$ & $225(26 \%)$ & \\
\hline Ulceration (No., $\mathrm{n}=1222$ ) & $434(36 \%)$ & $50(21 \%)$ & $384(39 \%)$ & $<.001$ \\
\hline Positive regional lymph node status (No., $n=1600$ ) & $424(27 \%)$ & $63(22 \%)$ & $361(27 \%)$ & .088 \\
\hline \multicolumn{5}{|l|}{ Pulmonary metastases (No.) } \\
\hline 1 & $473(28 \%)$ & $240(76 \%)$ & $233(17 \%)$ & $<.001$ \\
\hline 2 & $186(11 \%)$ & $49(15 \%)$ & $137(10 \%)$ & \\
\hline 3 & $75(4 \%)$ & $13(4 \%)$ & $62(4 \%)$ & \\
\hline$\geq 4$ & $986(57 \%)$ & $16(5 \%)$ & $970(69 \%)$ & \\
\hline \multicolumn{5}{|l|}{ Interval to pulmonary metastasis (No.) } \\
\hline$<1$ y & $395(23 \%)$ & $53(17 \%)$ & $342(25 \%)$ & $<.001$ \\
\hline $1-5 y$ & $911(53 \%)$ & $165(52 \%)$ & $746(53 \%)$ & \\
\hline$>5 y$ & $414(24 \%)$ & $100(31 \%)$ & $314(22 \%)$ & \\
\hline At least 1 other distant, nonpulmonary metastasis & $655(38 \%)$ & $61(19 \%)$ & $594(42 \%)$ & $<.001$ \\
\hline Chemotherapy* & $832(48 \%)$ & $99(31 \%)$ & $733(52 \%)$ & $<.001$ \\
\hline
\end{tabular}

Continuous and categorical variables were compared with the Student $t$-test, $\chi^{2}$ test, and $\mathrm{R} \times \mathrm{C}$ contingency tables when appropriate. *Chemotherapy administered at any time after initial diagnosis of primary lesion.

ification on number of preoperative risk factors identified in the multivariate analysis, patients with no risk factors, 1 risk factor, 2 risk factors, and 3 or more risk factors had 5-year survivals of $26 \%, 11 \%, 4 \%$, and $2 \%$, respectively (Figure 1).

Two hundred forty-nine (78\%) of the 318 patients who underwent metastasectomy had complete resection, and patients with incomplete resection were more likely to have multiple pulmonary metastases. Otherwise, there were no significant differences with regard to histologic type, diseases-free interval, or history of treated nonpulmonary distant metastasis (Table E1). Patients undergoing metastasectomy who had complete pathologic resection had a median survival of 19 months and a 5-year survival of $21 \%$, compared with a median survival of 11 months and a 5-year survival of only $13 \%$ for patients with incomplete resection 
TABLE 2. Independent predictors of overall survival in patients with pulmonary metastatic melanoma

\begin{tabular}{|c|c|c|c|}
\hline Predictor & Hazard ratio & 95\% Confidence interval & $P$ value \\
\hline Nodular histologic type & 1.1 & $1.01-1.3$ & .033 \\
\hline No. of pulmonary metastases $\geq 2$ & 1.2 & 1.04-1.4 & .012 \\
\hline Disease-free interval* & 1.5 & $(1.3,1.7)$ & $<.001$ \\
\hline \multicolumn{4}{|l|}{$<1 \mathrm{y}$} \\
\hline $1-5 y$ & 1.3 & $(1.1,1.4)$ & $<.001$ \\
\hline Presence of extrathoracic metastasis & 1.7 & $(1.6,1.9)$ & $<.001$ \\
\hline Pulmonary metastasectomy & 0.5 & $(0.4,0.6)$ & $<.001$ \\
\hline
\end{tabular}

*Reference category is disease-free interval longer than 5 years.

$(P<.0001$; Figure E5). Fifteen patients $(5 \%)$ underwent repeated surgical resections. The median time to repeated metastasectomy was $9 \pm 6.3$ months. There was no significant difference in survival between patients undergoing single metastasectomy and those patients undergoing repeated metastasectomies (median survivals 17 months and 15 months, respectively, $P=.9$ ).

\section{Discussion}

In this study, we found several independent prognostic factors to be associated with overall survival for patients with pulmonary metastatic melanoma. Patients who had nodular histologic type of the primary lesion, greater number of pulmonary metastases $(\geq 2)$, shorter disease-free interval $(<5$ years), or evidence of extrathoracic metastasis had a poor prognosis, with a median survival of less than 8 months. In contrast, patients selected for pulmonary metastasectomy were found to have a much higher median survival of 17 months. The greatest benefit from metastasectomy was realized in patients who had a prolonged diseasefree interval of greater than 5 years and no evidence of extrathoracic metastasis.

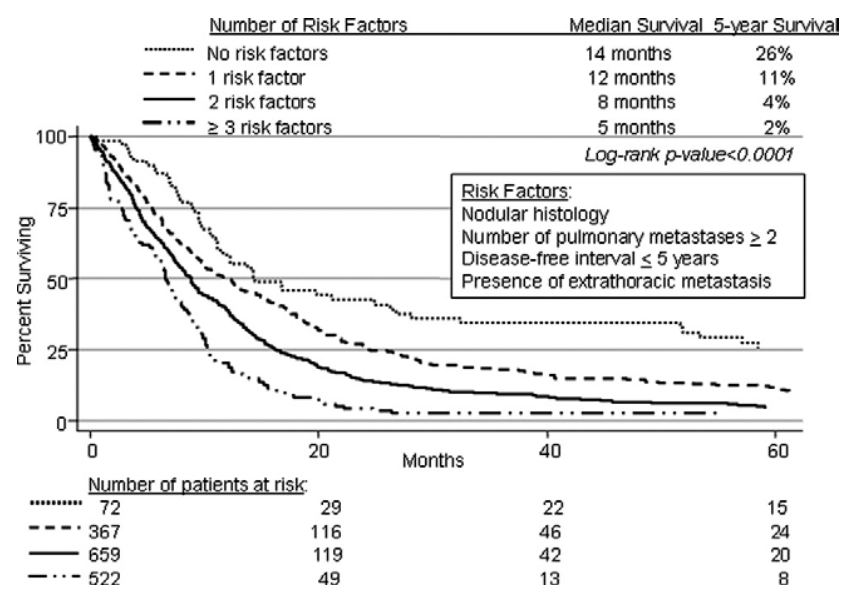

Figure 1. Overall survival by number of preoperative risk factors ( $\mathrm{n}=1720$ ).
Despite the many therapeutic advances that have been made during the past three decades for early-stage melanoma, the current treatment options for stage IV melanoma remain limited. Chemotherapy with dacarbazine remains the criterion standard, despite the lack of evidence of improved survival for patients with stage IV disease. ${ }^{11}$ In addition, there remains no role for immunotherapy in patients with metastatic melanoma. The reported response rates have been well below $20 \%$ for a variety of immunotherapies, including cytokines, monoclonal antibodies, and vaccination strategies with synthetic peptides, naked DNA, dendritic cells, recombinant viruses, and so on. ${ }^{12}$ Unfortunately, as a result of these dismal response rates and lack of phase 3 studies demonstrating improved overall survival for these systemic agents, surgery remains the only hope for improved survival for a select subset of patients with stage IV disease.

Previous observational studies of patients with metastatic melanoma have revealed varying results in identifying significant prognostic factors; however, several variables have remained constant from study to study, specifically the number of pulmonary nodules, the disease-free interval, and the presence of extrathoracic metastasis. ${ }^{2,9,10,13}$ In our previous analysis, performed more than 15 years ago, regional lymph node involvement and treatment with dacarbazinebased chemotherapy were found to be independent significant predictors of survival; however, both of these had questionable clinical significance, with a survival advantage of less than 2 months. ${ }^{9}$ In this larger analysis, these factors were not found to be significant predictors. The finding that chemotherapy was not associated with survival is consistent with randomized controlled trials investigating the efficacy of systemic therapy in this patient population. ${ }^{14-17}$

In contrast to systemic therapy, surgical management continues to be the primary treatment modality for patients with metastatic melanoma. Unfortunately, surgery is not appropriate for all patients, and patient selection criteria remain controversial. Although we did not directly determine predictors of survival among patients undergoing surgery, these patients typically had a limited number of lung 
nodules, no evidence of extrathoracic metastasis, and a prolonged disease-free interval.

All surgical patients included in this analysis underwent resection with curative intent only. None of the patients undergoing surgery in our series had grossly positive margins (R2). Incomplete resection in this study was defined as lack of evidence of gross residual disease; on further histologic examination, however, the margins of the specimen were judged to be microscopically incomplete (R1). This may explain why patients with "incomplete resection" in our series had a 5-year survival of $13 \%$, as compared with a $0 \%$ 5-year survival in the study reported by Leo and colleagues, ${ }^{10}$ in which incomplete resection was defined as either R1 (microscopically incomplete) or R2 (grossly incomplete). There is evidence to suggest that by reducing the tumor burden, the immune system's antitumor response may be enhanced. ${ }^{18}$ Together, these findings support the continued use of metastasectomy for select patients with melanoma and pulmonary metastasis.

The strengths of our study include prospective data collection, complete follow-up on all patients, and large sample size. Despite our attempts to control for many of the potential selection factors by accounting for clinical and pathologic characteristics of the metastasis, as well as characteristics of the individual patients, our analysis is most likely limited by patient selection bias. For example, it is challenging to account for the judgment of individual surgeons in determining eligibility for resection. In addition, we were unable to account for other comorbidities that also may have influenced the selection of patients for surgery and survival.

Although our study represents one of the largest series of pulmonary metastasectomies for melanoma, another limitation is that these data were collected during a span of 35 years. As a consequence, changes in imaging technology and less-invasive surgical techniques may also have influenced the selection and outcomes of patients. In an international registry of lung metastasis of 2988 patients with available preoperative radiologic and postoperative pathologic assessments, accuracy rates of $61 \%$ and $75 \%$ were reported for patients with bilateral and unilateral disease, respectively. In addition, radiologic assessment was found to underestimate number of pulmonary metastases by $16 \%$ to $25 \% .{ }^{13}$ Moreover, data from the recently completed National Lung Cancer Screening Trial $(\mathrm{n}=50,000)$ underscore the accuracy of newer generation chest CT scans that detect all lung abnormalities larger than $2 \mathrm{~mm}$. Most patients in the earlier phase of our series had their disease diagnosed by routine surveillance chest radiography alone; however, patients enrolled in the latter half of the study underwent CT, and more recently high-resolution helical CT with positron emission tomography (PET).

In addition, improvements in surgical technologies have led to an evolution of the standard approach for metasta- sectomy. Currently, we no longer routinely perform open thoracotomy at our institution, preferring limited-access (video-assisted) resections, either stapled wedge or lobectomy. This is accomplished by initially exploring the thorax thoracoscopically to inspect both the visceral and parietal pleural surfaces to exclude pleural dissemination. Pulmonary nodules for intended resection are then located with precise anatomic information from the CT scan and also by palpation of lesions with a long, oval-ringed clamp (thoracoscopic Foerster clamp). Occasionally, nodules in the anterior segments may also be palpated with the index finger through the anterior access incision. It is possible that these differences in operative technique may have also influenced patient selection for metastasectomy.

Surgical therapy for pulmonary metastasis is not a new treatment modality, as the first metastasectomy was performed more than 50 years ago, ${ }^{19}$ but controversy still exists regarding the benefits of resection for patients with stage IV melanoma. Several studies have demonstrated improved survivals of $18 \%$ to $39 \%$ for selected patients with pulmonary metastasis undergoing resection, which is a significant improvement relative to the 5-year survival of 3\% to 5\% for nonsurgically treated patients. ${ }^{2,6-10}$ In our study of 1720 patients, we also observed a significant survival benefit from pulmonary metastasectomy for a subset of patients with metastatic pulmonary melanoma. Our findings support the continued use of surgical resection for patients with the following criteria: (1) limited number of pulmonary metastases $(<2)$, (2) prolonged disease-free interval, and (3) negative results of PET or CT scan for extrathoracic pulmonary metastasis. A simple model that is based on number of preoperative risk factors is provided as an aid for clinicians when selecting patients appropriate for metastasectomy (Figure E3).

\section{References}

1. Jemal A, Tiwari RC, Murray T, Ghafoor A, Samuels A, Ward E, et al Cancer statistics, 2004. CA Cancer J Clin. 2004;54:8-29.

2. Essner R, Lee JH, Wanek LA, Itakura H, Morton DL. Contemporary surgical treatment of advanced-stage melanoma. Arch Surg. 2004;139: 961-7.

3. Balch CM, Soong SJ, Murad TM, Smith TW, Maddox WA, Durant JR A multifactorial analysis of melanoma. IV. prognostic factors in 200 melanoma patients with distant metastasis (stage III). J Clin Oncol. 1983;1:126-34.

4. Barth A, Wanek LA, Morton DL. Prognostic factors in 1521 melanoma patients with distant metastases. J Am Coll Surg. 1995;181:193201.

5. Lee ML, Tomsu K, Von Eschen KB. Duration of survival for disseminated malignant melanoma: results of a meta-analysis. Melanoma Res. 2000;10:81-92

6. Tafra L, Dale PS, Wanek LA, Ramming KP, Morton DL. Resection of adjuvant immunotherapy for melanoma metastatic to the lung and thorax. J Thorac Cardiovasc Surg. 1995;110:119-29.

7. Wong JH, Euhus DM, Morton DL. Surgical resection for metastatic melanoma of the lung. Arch Surg. 1988;123:1091-5.

8. Gorenstein LA, Putnam JB, Natarajan G, Balch CA, Roth JA. Improved survival after resection of pulmonary metastases from malignant melanoma. Ann Thorac Surg. 1991;52:204-10. 
9. Harpole DH, Johnson CM, Wolfe WG, George SL, Seigler HF. Analysis of 945 cases of pulmonary metastatic melanoma. J Thorac Cardiovasc Surg. 1992;103:743-50.

10. Leo F, Cagini L, Rocmans P, Cappello M, Van Geel A, Maggi G, et al. Lung metastases from melanoma: when is surgical treatment warranted? Br J Cancer. 2000;83:569-72.

11. Tarhini AA, Agarwala SS. Interleukin-2 for the treatment of melanoma. Curr Opin Investig Drugs. 2005;6:1234-9.

12. Riker AI, Jove R, Daud AI. Immunotherapy as part of a multidisciplinary approach to melanoma treatment. Front Biosci. 2006;11:1-14.

13. Pastorino U, Buyse M, Friedel G, Ginsberg RJ, Girard P, Goldstraw P, et al. Long-term results of lung metastasectomy: prognostic analyses based on 5206 cases. J Thorac Cardiovasc Surg. 1997;113:37-49.

14. Atkins MB, Lotze MT, Dutcher JP, Fisher RI, Weiss G, Margolin K, et al. High-dose recombinant interleukin 2 therapy for patients with metastatic melanoma: analysis of 270 patients treated between 1985 and 1993. J Clin Oncol. 1999;17:2105-16.

15. Atkins MB, Kunkel L, Sznol M, Rosenberg SA. High-dose recombinant interleukin-2 therapy in patients with metastatic melanoma: longterm survival update. Cancer J Sci Am. 2000;6 Suppl 1):S11-4.

16. Mandara M, Nortilli R, Sava T, Cetto GL. Chemotherapy for metastatic melanoma. Expert Rev Anticancer Ther. 2006;6:121-30.

17. Alexandrescu DT, Dutcher JP, Wiernik PH. Metastatic melanoma: is biochemotherapy the future? Med Oncol. 2005;22:101-10.

18. Dreno B, Nguyen JM, Khammari A, Pandolfino MC, Tessier MH, Bercegeay S, et al. Randomized trial of adoptive transfer of melanoma tumor-infiltrating lymphocytes as adjuvant therapy for stage III melanoma. Cancer Immunol Immunother. 2002;51:539-46.

19. Oschner A, Rush V. Treatment of pulmonary metastatic disease. Surg Clin North Am. 1966;46:1469-74.

\section{Discussion}

Dr Joshua Robert Sonett (New York, NY). Dr Petersen, I congratulate you and your colleagues on a nicely presented, well written, and, I believe, important article. The Duke oncology database has enabled the largest review of a single-center result of pulmonary metastasectomy in melanoma. Importantly, the database includes both nonsurgical and surgical patients in the cohort. The primary variable analysis was performed on 318 patients undergoing resection, a carefully selected cohort representing $18 \%$ of all patients with pulmonary metastasis in your center. The results of the analysis are reassuring, nicely corroborating the previous work presented by Ugo Pastorini of the international registry of lung metastases, a collaboration of US, Canadian, and European centers. That collaborative registry was created to facilitate the study of results of pulmonary metastasectomy and to divine prognostic variables to guide treatment, much as your study was. When the data were presented in 1997 with 5000 cases, 282 represented patients with melanoma who underwent curative resection. Overall survival of resected patients in that study was $21 \%$ at 5 years. And although patients with melanoma histologic type had the worst prognosis of all those with solid tumors in that review, the nearly identical 5-year survival results presented today clearly confirm and validate the utility of pulmonary metastasectomy for select patients with metastatic melanoma. Additionally, the international registry reported a follow-up study of 328 patients in which four groups of risk-stratified variables were defined to help set the standards for delineating the role of metastasectomy in melanoma. Again, these results and stratifications are nicely similar to your reported results, with the additional risk factors that you added of nodular histologic type and extrathoracic metastasis. That brings me to my first question.

Classically and sensibly, pulmonary metastasectomy with curative intent is reserved for patients without any evidence of ex- trathoracic disease. In your series, $20 \%$ of your patients undergoing resection were defined as having other distant metastases. Please explain why you brought these patients to resection and how you would deal with them in the future.

Two, given the similar results of the prognostic groups in the international registry data, how do you think the addition of nodular histologic type helps us to decide which patients with metastatic melanoma should undergo pulmonary resection or the clinical timing of the potential resection?

And finally, you noted that in the age of improved imaging your group preferentially now uses video-assisted thoracoscopic surgical techniques for pulmonary metastasectomy. I wholeheartedly support this approach, but historically video-assisted thoracoscopic surgical pulmonary metastasectomy has been considered controversial. Can you comment on this?

Again, I congratulate you on a well done and well presented study, and thank the Association for the privilege of discussing this presentation.

Dr Petersen. Thank you, Dr Sonett, for your insightful comments and questions.

With regard to your first question about our $19 \%$ rate of extrathoracic metastasis, all such patients who underwent surgery either had a history of previous extrapulmonary resection or underwent concurrent extrapulmonary resection. Although the rate seems high at $19 \%$, it is similar to the rate reported by Leo and colleagues in the subset analysis of patients with melanoma only of the international registry of lung metastases. The larger study by Pastorino and colleagues, which included a variety of tumor types, actually showed a trend toward worse prognosis for patients with previous or concurrent extrapulmonary resections, which is consistent with the findings in our study.

With regard to your question of how to use some of these predictors that we have discriminated in our multivariate model, such as nodular histologic type or disease-free interval, one of the figures that we presented revealed varying survival curves when stratifying by number of preoperative risk factors. We believe it is important to take into consideration all risk factors when deciding whether the patient should undergo surgery. For instance if a patient had nodular histologic type of the primary tumor but had a prolonged disease-free interval, a limited number of pulmonary metastases, and no evidence of extrathoracic disease, we would not be dissuaded from surgery but would inform the patient of the slightly higher mortality relative to patients without that risk factor.

With regard to your last question about thoracoscopic surgery, it is true that this subject remains controversial. We realize this is not standard of practice at many other institutions. Of the 318 patients with metastasis who underwent surgical resection in our series, 40 underwent resection by a thoracoscopic approach. All these patients were evaluated preoperatively by combined PET-CT scan, resulting in a low likelihood of having missed lesions larger than $3 \mathrm{~mm}$. The sensitivity of finding pulmonary metastasis from melanoma by combined PET-CT scan has been previously reported by Finkelstein and colleagues to be around $88 \%$, with a specificity of $91 \%$. The worst-case scenario for the small number of patients with missed lesions not found by preoperative imaging would be to undergo a sequential thoracoscopic resection. Moreover, there is no evidence to date that these missed lesions, found 
intraoperatively by manual palpation through an open approach and probably measuring on the order of $3 \mathrm{~mm}$ or less, given the newest generation of high-resolution CT imaging and combination PET-CT scan, are of any detriment to the patient.

Dr Mark J. Krasna (Baltimore, Md). Excellent presentation and good answers to those questions. Just one quick comment. In both colon cancer and renal cell carcinoma, we now have data on the importance of lymph node metastasis. At many centers, we have actually begun to implement routine mediastinoscopy before attempting a curative metastasectomy. Although it only panned out in your univariate analysis and did not show up in the Cox regression analysis, would you comment on how you evaluate the mediastinum in these patients with metastatic melanoma before they are taken to metastasectomy?

Dr Petersen. The regional lymph node status that showed up as a significant predictor in our univariate analysis had to do with the primary tumor only. The significance of positive mediastinal lymph nodes to which you are alluding to remains unclear, however, especially in the setting of metastatic disease. Unfortunately, this will never be answered unless lymph node dissection in patients with pulmonary metastatic melanoma is uniformly practiced. A maximum of only $9 \%$ of patients in the international registry of lung metastases underwent mediastinal lymph node dissection. Because currently most patients with suspected pulmonary metastatic melanoma undergo wide margin wedge resection only without previous mediastinoscopy or concurrent lymph node dissection, this question will remain largely unanswered until we have a known denominator.
Dr Frank C. Detterbeck (New Haven, Conn). I just want to follow up on Dr Sonett's comment about video-assisted resection. We and others have shown that you still need to do palpation, even with helical CT, because approximately $20 \%$ to $25 \%$ of patients will have additional metastases found on palpation despite helical CT. We have done a follow-up study trying to look at factors that might have predicted that. Was it that the radiologists were undercalling it? We have not been able to identify factors. So I think that if you are trying to do a complete resection, you still need to palpate.

You must have decided that you weren't going to try to do a complete resection; you were just going to see how many patients had follow-up nodules. Have you gone back and actually looked at that to provide some data on that?

Dr Petersen. No, we have not specifically looked at that question in the metastasectomy series, but that is a very good point and something that we can look into in the future. The patients in our study that underwent thoracoscopic resection were evaluated preoperatively with the latest generation of high-resolution CT and combined PET-CT. It is becoming standard practice at our institution to evaluate these patients with combined PET-CT, which as we have previously discussed has high sensitivity and specificity, making it less likely to miss lesions larger than $3 \mathrm{~mm}$. Unfortunately, to date there are no data to support that going after all of the very small lesions that may or may not be found by aggressive intraoperative manual palpation through an open approach improves overall prognosis. In fact, the likelihood of false positives increases the smaller the lesion is, and by aggressively resecting all these, you may actually be jeopardizing pulmonary reserve; however, this remains largely unknown. 


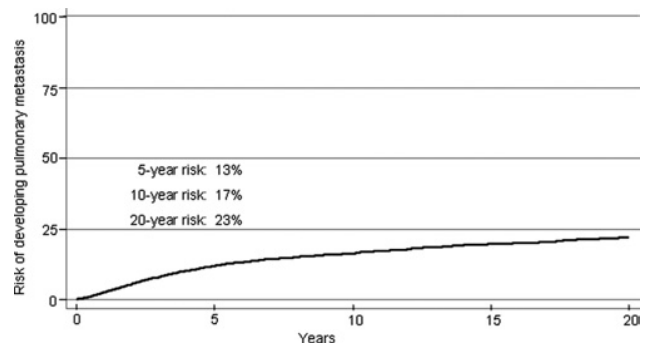

Figure E1. Risk of development of pulmonary metastasis ( $\mathrm{n}=$ 13,565).

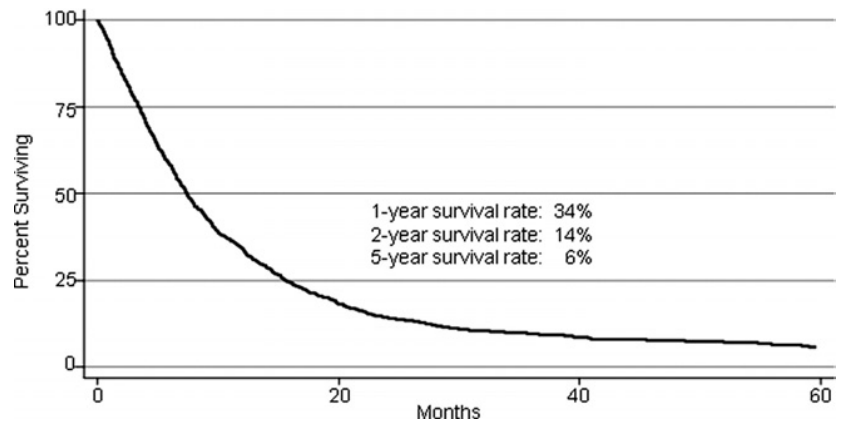

Figure E2. Overall survival for patients with metastatic pulmonary melanoma $(n=1720)$.

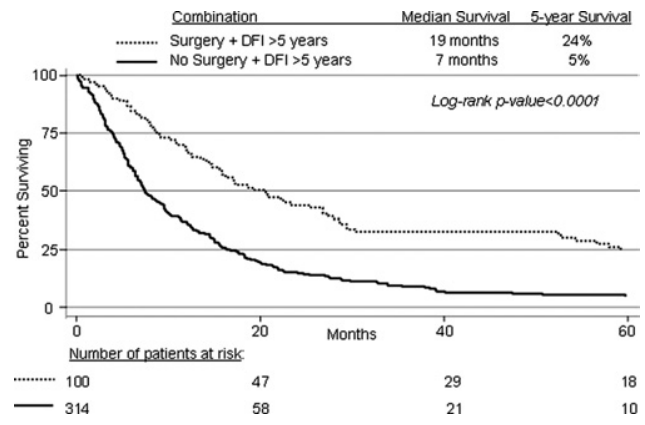

Figure E3. Overall survival by metastasectomy and disease-free interval ( $\mathrm{n}=414)$.

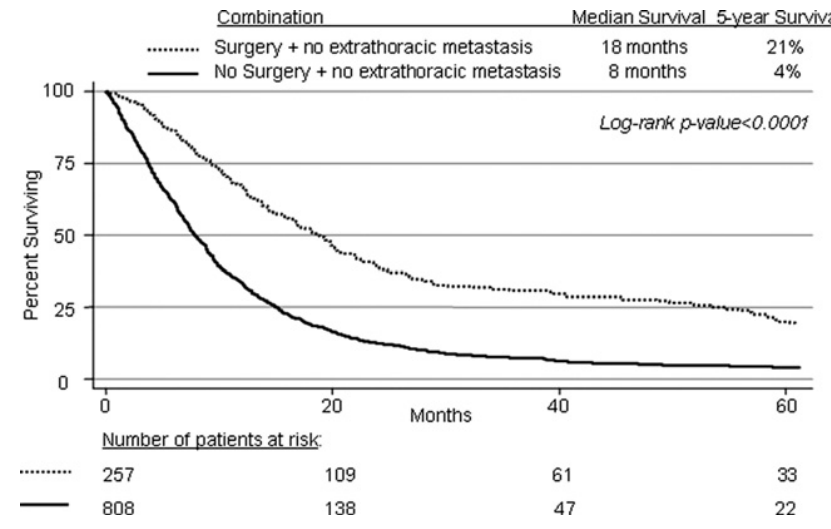

Figure E4. Overall survival by metastasectomy and presence of extrathoracic metastasis $(n=1065)$.

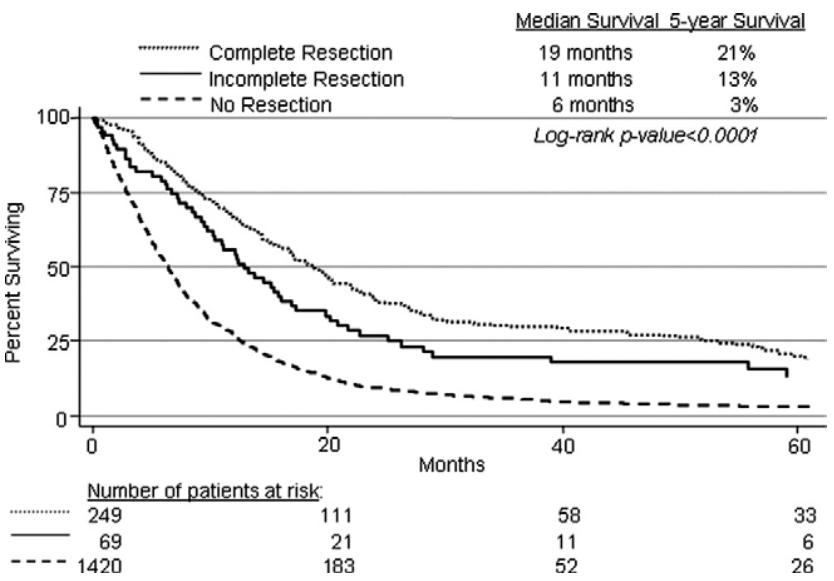

Figure E5. Overall survival by complete resection, incomplete resection, and nonsurgical treatment $(n=1720)$. 
TABLE E1. Surgery-only patients, by complete and incomplete resection $(\mathrm{n}=\mathbf{3 1 8}$ )

\begin{tabular}{|c|c|c|c|}
\hline Risk factor & Complete $(n=249)$ & Incomplete $(n=69)$ & $P$ value \\
\hline \multicolumn{4}{|l|}{ Histologic type (No.) } \\
\hline Nodular & $47(19 \%)$ & $12(20 \%)$ & .78 \\
\hline Superficial spreading & $95(38 \%)$ & $26(38 \%)$ & .94 \\
\hline Lentigo maligna & $18(7 \%)$ & $3(4 \%)$ & .40 \\
\hline Other, not otherwise specified & $82(33 \%)$ & $28(40 \%)$ & .24 \\
\hline 2 & $24(10 \%)$ & $25(36 \%)$ & \\
\hline 3 & $4(1.5 \%)$ & $9(13 \%)$ & \\
\hline$\geq 4$ & $1(0.5 \%)$ & $15(22 \%)$ & \\
\hline Interval to pulmonary metastasis (No.) & & & 0.33 \\
\hline$<1 \mathrm{y}$ & $44(18 \%)$ & $9(13 \%)$ & \\
\hline
\end{tabular}

